\title{
A comparison of the local spiral structure from Gaia DR2 and VLBI maser parallaxes ${ }^{\star}$
}

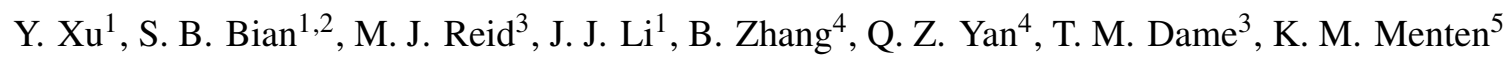 \\ Z. H. He ${ }^{1}$, S. L. $\mathrm{Liao}^{4}$, and Z. H. Tang ${ }^{4}$ \\ 1 Purple Mountain Observatory, Nanjing 210008, PR China \\ e-mail: xuye@pmo.ac.cn \\ 2 Shanghai Normal University, Shanghai 200234, PR China \\ 3 Harvard-Smithsonian Center for Astrophysics, 60 Garden Street, Cambridge, MA 02138, USA \\ ${ }^{4}$ Shanghai Observatory, Shanghai 200030, PR China \\ 5 Max-Planck-Institut für Radioastronomie, Auf dem Hügel 69, 53121 Bonn, Germany
}

Received 10 May 2018 / Accepted 24 June 2018

\begin{abstract}
Context. The Gaia mission has released the second data set (Gaia DR2), which contains parallaxes and proper motions for a large number of massive, young stars.

Aims. We investigate the spiral structure in the solar neighborhood revealed by Gaia DR2 and compare it with that depicted by VLBI maser parallaxes.

Methods. We examined three samples with different constraints on parallax uncertainty and distance errors and stellar spectral types: (1) all OB stars with parallax errors of less than 10\%; (2) only O-type stars with 0.1 mas errors imposed and with parallax distance errors of less than $0.2 \mathrm{kpc}$; and (3) only O-type stars with 0.05 mas errors imposed and with parallax distance errors of less than $0.3 \mathrm{kpc}$. Results. In spite of the significant distance uncertainties for stars in DR2 beyond $1.4 \mathrm{kpc}$, the spiral structure in the solar neighborhood demonstrated by Gaia agrees well with that illustrated by VLBI maser results. The O-type stars available from DR2 extend the spiral arm models determined from VLBI maser parallaxes into the fourth Galactic quadrant, and suggest the existence of a new spur between the Local and Sagittarius arms.
\end{abstract}

Key words. Galaxy: structure - stars: early-type - stars: massive - masers

\section{Introduction}

The Milky Way has been thought to be a spiral galaxy since as far back as the 1850s (Alexander 1852), but it is extremely difficult to observe its spiral structure due to our edge-on view from its interior and limited by copious dust extinction. It was not until the 1950s that researchers started to use high-mass stars (OB stars) and $\mathrm{H}_{\text {II }}$ regions to trace spiral arm segments in the solar neighborhood (Morgan et al. 1952, 1953). Such work, augmented by radio wavelength data, was extended to cover most of the Galaxy by Georgelin \& Georgelin (1976) and Russeil (2003). In the last decade, Very Long Baseline Interferometry (VLBI) at centimeter wavelengths has provided hundreds of parallaxes to very young, massive stars with accuracies often as good as \pm 0.01 mas (Xu et al. 2006, 2016; Honma et al. 2007; Reid et al. 2014), substantially increasing our knowledge of the spiral arms in our Galaxy. Recent VLBI parallaxes, however, have mostly been limited to stars visible from the northern hemisphere, leaving the fourth Galactic quadrant largely unexplored.

The Gaia satellite (Gaia Collaboration 2016), launched in 2013, will ultimately achieve parallax accuracy comparable to that of VLBI for approximately $10^{9}$ stars, although the current

* The full Table 1 is only available at the CDS via anonymous ftp to cdsarc.u-strasbg.fr $(130.79 .128 .5)$ or via http://cdsarc.u-strasbg.fr/viz-bin/qcat?J/A+A/616/L15 release (Gaia Collaboration 2018) is limited by systematic error of up to $<0.1$ mas, depending on celestial positions, magnitudes, and colors (Lindegren et al. 2018). Still, even with this limitation, distances to many OB stars within a couple of kiloparsec from the Sun are now available. We note that even when Gaia reaches its target accuracy, direct measurement of spiral structure will be limited by dust extinction in the plane of the inner Galaxy to stars typically within a few kiloparsec.

\section{OB star sample}

Owing to their short main sequence lifetimes, high-mass stars are generally located near their birth places, and consequently can trace Galactic spiral structure. However, due to the absence of effective temperatures (Gaia Collaboration 2018) for bright stars $(>10000 \mathrm{~K})$ in Gaia DR2, we are unable to identify OB stars straightforwardly. In order to identify OB stars, we started with a sample of them from the Catalog of Galactic OB Stars (Reed 2003), which gives coordinates accurate to $\approx 1^{\prime \prime}$, along with stellar classifications and Heliocentric radial velocities from SIMBAD ${ }^{1}$. We then cross-matched these stars with the Gaia DR2 catalog using a match radius of $1^{\prime \prime}$. After having eliminated those with multiple matches, 5772 O-B2 stars remain (see Table 1). Our analysis starts with this sample.

\footnotetext{
1 http://simbad.u-strasbg.fr/simbad/sim-fcoo
} 
Table 1. Parallaxes and proper motions of OB stars.

\begin{tabular}{|c|c|c|c|c|c|c|c|c|}
\hline Name & Gaia DR2 ID & $\begin{array}{l}\text { RA } \\
\left({ }^{\circ}\right)\end{array}$ & $\begin{array}{l}\text { Dec } \\
\left({ }^{\circ}\right)\end{array}$ & $\begin{array}{l}\pi \\
\text { (mas) }\end{array}$ & $\begin{array}{l}\mu_{x} \\
\left(\operatorname{mas~yr}^{-1}\right)\end{array}$ & $\begin{array}{l}\mu_{y} \\
\left(\operatorname{mas~yr}^{-1}\right)\end{array}$ & $\begin{array}{l}V_{\mathrm{LSR}} \\
\left(\mathrm{km} \mathrm{s}^{-1}\right)\end{array}$ & $\begin{array}{l}\text { Spectral } \\
\text { type }\end{array}$ \\
\hline ALS 13358 & 528563384392653312 & 0.1219 & 67.2168 & $1.079 \pm 0.051$ & $-1.72 \pm 0.07$ & $-2.39 \pm 0.08$ & & B1 \\
\hline ALS 13363 & 423149081478526464 & 0.2867 & 58.9799 & $0.302 \pm 0.030$ & $-3.26 \pm 0.05$ & $-2.62 \pm 0.04$ & & B0 \\
\hline ALS 13366 & 423157774492279680 & 0.3113 & 59.1428 & $0.305 \pm 0.028$ & $-3.04 \pm 0.04$ & $-1.34 \pm 0.04$ & & B2 \\
\hline ALS 13367 & 429338850909909760 & 0.3485 & 60.3555 & $0.950 \pm 0.054$ & $1.94 \pm 0.09$ & $-3.82 \pm 0.07$ & -5.2 & B2ne \\
\hline ALS 13375 & 528594342521399168 & 0.4453 & 67.5070 & $1.016 \pm 0.031$ & $-1.57 \pm 0.04$ & $-1.77 \pm 0.04$ & & $09.5 \mathrm{~V}$ \\
\hline
\end{tabular}

Notes. Column 1 is the OB star identity in the catalog of Reed (2003); Column 2 is the unique source identifier in Gaia DR2. Columns 3 and 4 are barycentric right ascension (RA) and declination (Dec). Columns 5-7 give the parallax and proper motion in the eastward $\left(\mu_{x}=\mu_{\alpha} \cos \delta\right.$ ) and northward directions $\left(\mu_{y}=\mu_{\delta}\right.$ ). Column 8 lists local standard of rest (LSR) velocity converted from the heliocentric value. Column 9 is the spectral type of the stars. In Gaia DR2, the reference epoch is J2015.5. The full table is available at the CDS.

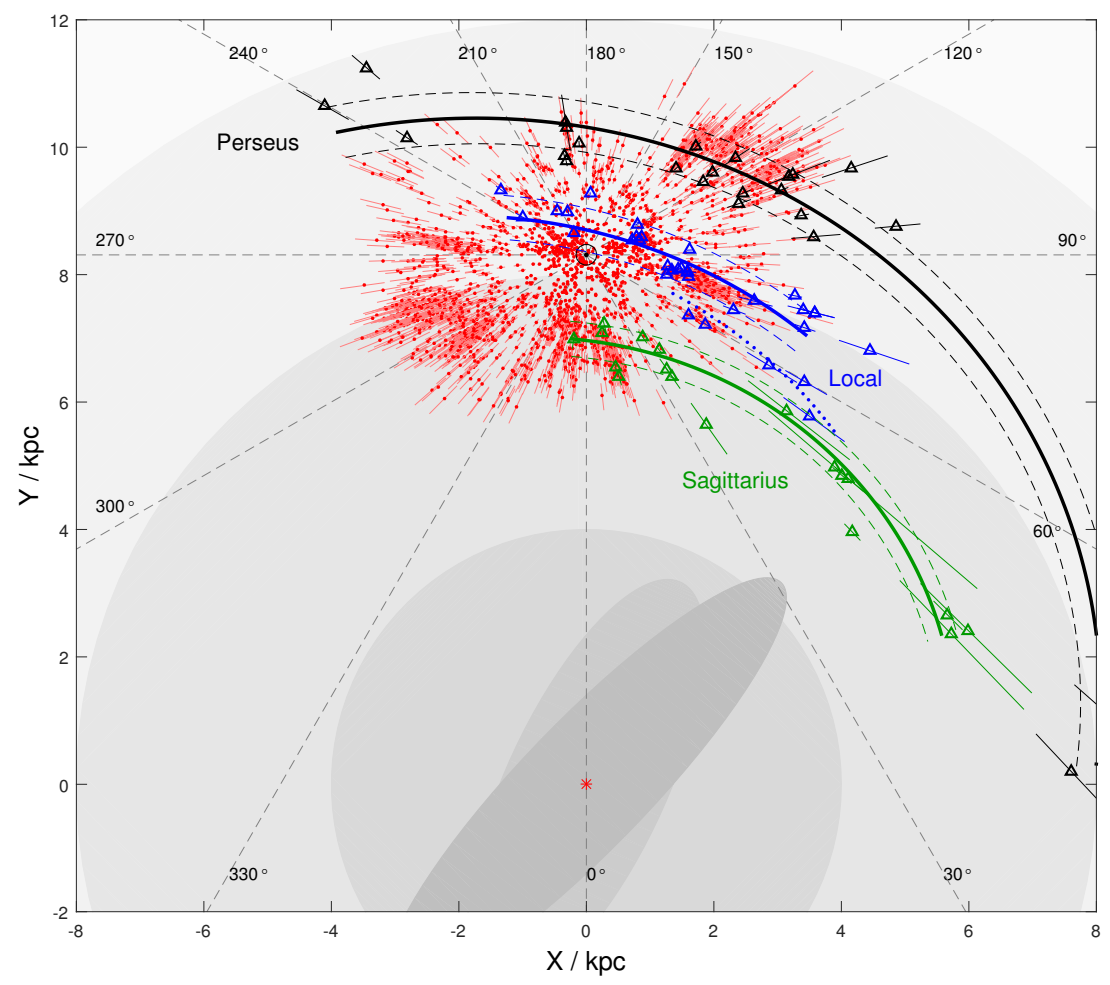

Fig. 1. Locations of Gaia DR2 OB (O-B2) stars (red circles) and masers (triangles) (Reid et al. 2014; Xu et al. 2016). The formal parallax uncertainties of the OB stars shown here are better than $10 \%$, but do not include a possible \pm 0.1 mas systematic error. The Galactic center (red star) is at $(0,0)$ and the Sun (Sun symbol) is at $(0,8.31)$. The solid and dashed curved lines denote the center and $\pm 1 \sigma$ widths of spiral arm models based on VBLI maser parallaxes. The Perseus arm (black), the Local arm (blue), and Sagittarius arm (green) are shown. Straight dashed lines indicate Galactic longitude.

Following the recommendation of the Gaia team (Arenou et al. 2018), we have not corrected the parallaxes of the individual OB stars in our sample by the average systematic bias of 0.029 mas on the parallax zero-point. Such a correction would, in any case, be very small for the nearby $\mathrm{OB}$ stars that we examine here. Table 1 lists coordinates, parallaxes, proper motions, radial velocities, and classifications of these OB stars. We believe this is the largest sample of O-B2 stars with parallaxes and proper motions to date.

\section{Spiral structure}

In this section, we investigate the spiral structure of the Milky Way within $\approx 3 \mathrm{kpc}$ as revealed by OB stars and compare it with that determined from maser parallaxes associated with extremely young $\left(<10^{5} \mathrm{yr}\right)$ high-mass star forming regions, whose parallaxes have been measured accurately with VLBI. Most of the stars in the sample have parallax uncertainties larger than $\pm 20 \%$, which can lead to distance uncertainty comparable to the spacing between spiral arms. Therefore, only stars with parallax accuracies better than $\pm 10 \%$ are adopted, yielding a reduced sample of 2800 sources. We plot the locations of these stars in Fig.1, along with stars with VLBI parallaxes from Reid et al. (2014) and Xu et al. (2016), which extend much further into the Galaxy.

As expected, most of the Gaia OB stars in our reduced sample gather around the Sun within a radius of about $3 \mathrm{kpc}$, as distant OB stars have been rejected owing to their large distance uncertainties and/or extinctions. The Gaia data generally 


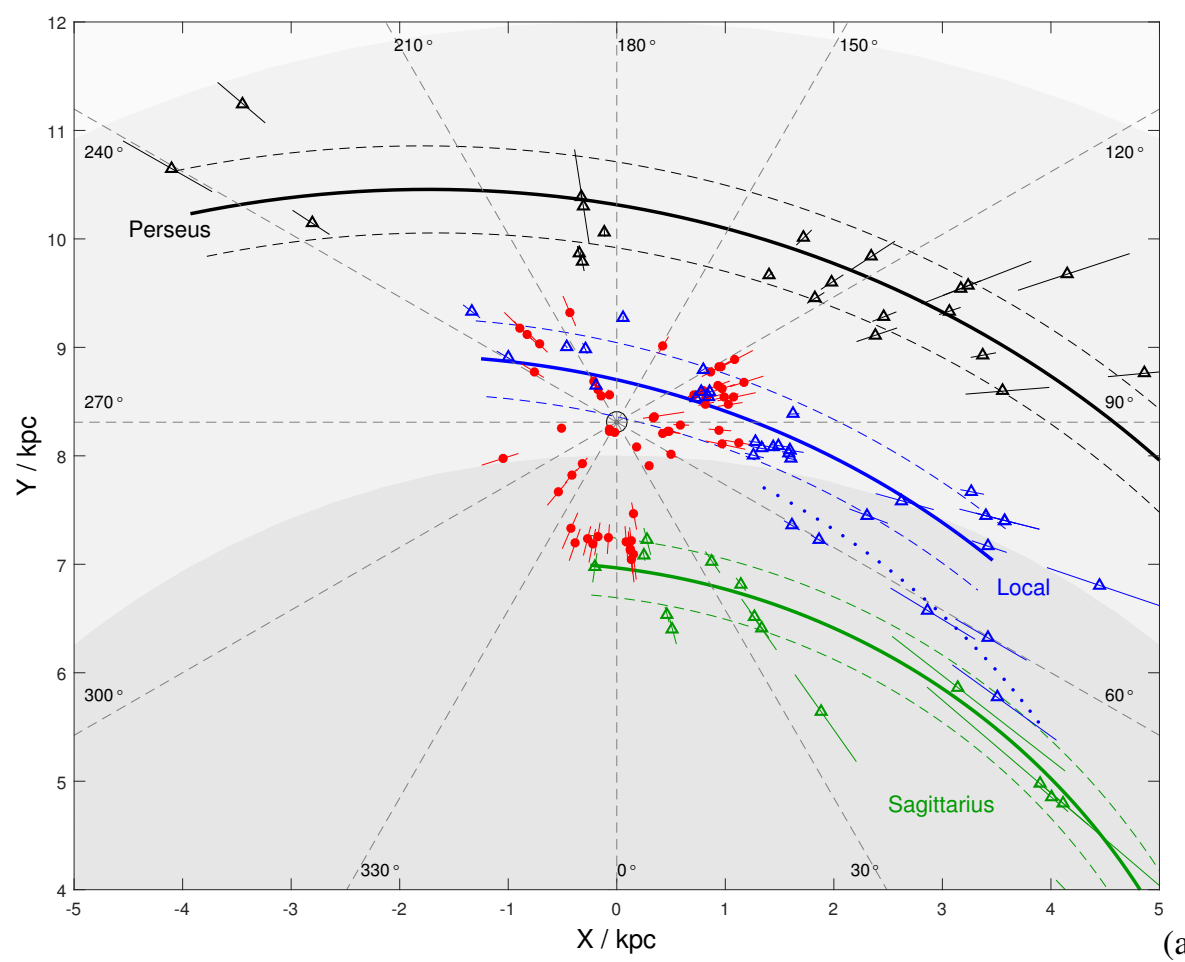

(a)

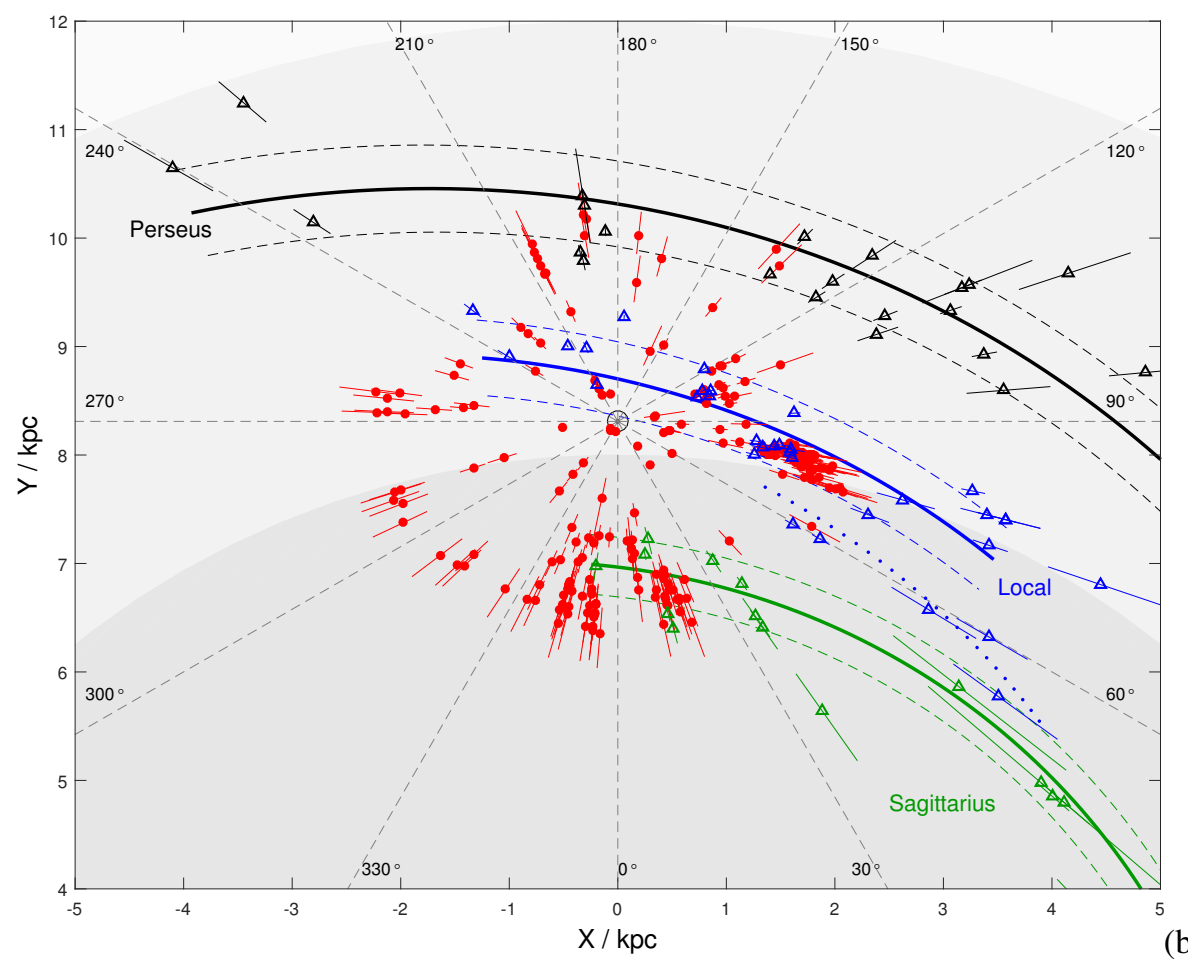

Fig. 2. Locations of only the identified O-type stars in Gaia DR2 (red circles): panel a) with \pm 0.1 mas systematic errors added in quadrature to the formal DR2 errors; only stars with corresponding absolute distance uncertainties $<0.2 \mathrm{kpc}$ are shown; Panel $b$ ) with \pm 0.05 mas systematic errors added in quadrature to the formal DR2 errors; only stars with corresponding absolute distance uncertainties $<0.2 \mathrm{kpc}$ are shown. See Fig. 1 caption for other details, but note that the $10 \%$ error threshold in that figure is not used here.

confirm the locations of spiral arms from VLBI in the Perseus, Local, and Sagittarius arms. Between Galactic longitude $240^{\circ}$ and $360^{\circ}$, they can be used to extend our knowledge of the latter two arms.

As is also apparent in Fig. 1, OB stars presumably at nearly the same distance are spread out along the line of sight, suggesting that the $10 \%$ threshold for parallax uncertainties is insufficient at distances beyond about $1.4 \mathrm{kpc}$. In addition, a substantial number of OB stars appear to fall between spiral arms. Since this is rarely the case for the maser sample of very young and massive stars, it suggests that allowing stars as late as type B2 into the sample is blurring spiral structure. These stars can 
live long enough to orbit roughly half the way around the Galaxy and may have left the spiral arms.

In order to obtain an OB star sample that is truly capable of tracing spiral arms, we start over from our initial sample of 5772 stars, abandoning the $10 \%$ uncertainty constraint and removing B-type stars. For the remaining O-type stars, we incorporate the systematic error 0.1 mas on parallaxes recommended by Lindegren et al. (2018) and require absolute distance uncertainty $<0.2 \mathrm{kpc}$ and peculiar motions $<20 \mathrm{~km} \mathrm{~s}^{-1}$ (the calculations of peculiar motions are given in the appendix). This leaves a sample of 59 O-type stars. These stars of O9.7V or earlier should have main sequence lifetimes of $<8$ Myr (Weidner \& Vink 2010), and with peculiar motions $<20 \mathrm{~km} \mathrm{~s}^{-1}$, they should not have moved more than $0.2 \mathrm{kpc}$ from their birthplaces. Because the widths of the spiral arms neighboring the Sun are $\approx 0.3 \mathrm{kpc}$ (Reid et al. 2014) and lines of sight usually are not perpendicular to spiral arms, these stars should still be in their birth spiral arms.

In Fig. 2a, we display the distribution of those 59 O-type stars. Most of them are consistent with being in the Local and Sagittarius spiral arms, as traced by masers. Interestingly, about 10 of the stars appear to be between these two arms. This observation should be reliable as it comes from a very conservative treatment of the Gaia DR2 parallaxes.

If we relax the assumed DR2 systematic parallax uncertainty from \pm 0.1 to \pm 0.05 mas and loosen the absolute distance error constraint from $\pm 0.2 \mathrm{kpc}$ to $\pm 0.3 \mathrm{kpc}$, the sample increases to 241 stars and is displayed in Fig. 2b. Significant linear arrangements that point toward the Sun ("fingers of God") are now more apparent, suggesting that caution should be exercised when gleaning information about spiral structure from this sample. However, some interesting features, which are not strongly dependent on the distance uncertainty, can be seen.

The abundance of stars between about $240^{\circ}$ and $270^{\circ}$ longitude at distances $>1 \mathrm{kpc}$ suggest that the Local arm continues into the fourth quadrant and bends somewhat inward toward the Galactic center. Similarly, the stars between $300^{\circ}$ and $320^{\circ}$ longitude at distances $>1 \mathrm{kpc}$ extend the Sagittarius arm into the fourth quadrant with about the same pitch angle as that determined from the maser parallaxes. Finally, a small cluster of stars centered near longitude $\approx 290^{\circ}$ at a distance of $\approx 2 \mathrm{kpc}$ appear to extend upward from the Sagittarius arm at a fairly high angle, as one might expect for a Galactic spur. This should not be surprising as the maser parallaxes have already shown some spurs; for example, the Aquila spur (Cohen et al. 1980; Dame et al. 1986).

\section{Conclusions}

VLBI parallaxes have traced the Milky Way's spiral structure within about $10 \mathrm{kpc}$ of the Sun in Galactic quadrants 1, 2, and 3. The Gaia DR2 data supplement the maser VLBI measurements and extend the map of the Local and Sagittarius arms into quadrant 4 . They also suggest an additional spur-like feature between those arms.

Acknowledgements. This work was supported by the National Natural Science Foundation of China (Grant Numbers: 11673066, U1431227, 11673051, $11703065,11573054,11503042)$, the Natural Science Foundation of Shanghai under grant 15ZR1446900, and the 100 Talents Project of the Chinese Academy of Sciences. This work has made use of data from the European Space Agency (ESA) mission Gaia (https://www.cosmos.esa.int/gaia), processed by the Gaia Data Processing and Analysis Consortium (DPAC, https://www. cosmos.esa.int/web/gaia/dpac/consortium). Funding for the DPAC has been provided by national institutions, in particular the institutions participating in the Gaia Multilateral Agreement.

\section{References}

Alexander, S., 1852, AJ, 2, 95

Arenou, F., Luri, X., Babusiaux, C., et al. 2018, A\&A, 616, A17

Cohen, R. S., Cong, H., Dame, T. M., \& Thaddeus, P. 1980, ApJ, 239, L53

Dame, T. M., Elmegreen, B. G., Cohen, R. S., \& Thaddeus, P. 1986, ApJ, 305, 892

Gaia Collaboration (Prusti, T., et al.) 2016, A\&A, 595, A1

Gaia Collaboration (Brown, A. G. A., et al.) 2018, A\&A, 616, A1

Georgelin, Y. M., \& Georgelin, Y. P. 1976, A\&A, 49, 57

Honma, M., Bushimata, T., Choi, Y. K., et al. 2007, PASJ, 59, 889

Lindegren, L., Hernandez, J., Bombrun, A., et al. 2018, A\&A, 616, A2

Morgan, W. W., Sharpless, S., \& Osterbrock, D. 1952, AJ, 57, 3

Morgan, W. W., Whitford, A. E., \& Code, A. D. 1953, ApJ, 118, 318

Reed, B. C. 2003, AJ, 125, 2531

Reid, M. J., Menten, K. M., Zheng, X. W., et al. 2009, ApJ, 700, 137

Reid, M. J., Menten, K. M., Brunthaler, A., et al. 2014, ApJ, 783, 130

Russeil, D. 2003, A\&A, 397, 133

Weidner, C., \& Vink, J. S. 2010, A\&A, 524, A98

Xu, Y., Reid, M. J., Zheng, X. W., \& Menten, K. M. 2006, Science, 311, 54

Xu, Y., Reid, M., Dame, T., et al. 2016, Sci. Adv., 2, e1600878

\section{Appendix A: The calculation of the peculiar motions of $O$ stars}

With distances, proper potions, and radial velocities, one has full three-dimensional (3D) velocity information in a heliocentric reference frame. Adding the Sun's full motion with respect to a reference frame at rest at the Galactic center transfers the O-star motions to that frame. Then removing a model of pure circular motion gives Galactic peculiar motions, expressed by $\left(U_{\mathrm{s}}, V_{\mathrm{s}}, W_{\mathrm{s}}\right)$, which are velocity components toward the Galactic center (GC), in the direction of Galactic rotation, and toward the north Galactic Pole at the location of each star.

We estimate peculiar motions of the $O$ stars following Reid et al. (2009), using updated Galactic parameters of $241 \mathrm{~km} \mathrm{~s}^{-1}$ for the Galactic rotation speed, $\Theta_{0}$, at a distance of $8.31 \mathrm{kpc}$ to the GC, $R_{0}$, and solar motion parameters of $U_{\odot}=10.5 \mathrm{~km} \mathrm{~s}^{-1}, V_{\odot}=14.4 \mathrm{~km} \mathrm{~s}^{-1}$, and $W_{\odot}=8.9 \mathrm{~km} \mathrm{~s}^{-1}$ from Reid et al. (2014). We used the "Universal" rotation curve model, which produces a relatively flat rotation curve between 5 and $15 \mathrm{kpc}$. 